Viewpoint

\title{
A century of sensory processing dysfunction in schizophrenia
}

\section{Introduction}

Approximately 100 years ago, Bleuler famously declared that "Sensory response to external stimulus is quite normal" in schizophrenia [1], followed however by the cryptic statement: "Busch and Kraepelin have found in perception experiment (using the shutter and revolving drum apparatus) that schizophrenics show many more errors and particularly omissions than do the healthy ... . Using accurate apparatus, we were unable to substantiate these findings" [1].

Bleuler's statements led to a century of formulations of schizophrenia in which perceptual deficits were de-emphasized relative to other types of cognitive operations, including the wellknown four" A"s of Bleuler (i.e., [disturbance of] affect, association, ambivalence and autism). This viewpoint addresses the concept of sensory/perceptual dysfunction in schizophrenia, and reconstructs the Busch/Kraepelin experiment alluded to by Bleuler. We argue that with the benefit of hindsight, the findings of Busch/Kraepelin were probably not only correct but also prescient, and that sensory deficits remain a critical, albeit underemphasized, feature of schizophrenia. As in the time of Kraepelin and Bleuler, technical issues as well as theoretical formulations may still be contributing to the de-emphasis of sensory/perceptual deficits vs. other aspects of cognitive impairment in schizophrenia.

\section{Early history of sensory studies in schizophrenia}

\subsection{Visual sensory deficits}

When Kraepelin first described the syndrome of Dementia Praecox in the late 1800 s, he believed that genetically based generalized processes, such as autointoxication, led to the clinical manifestations. Subsequently, Kraepelin characterized Dementia Praecox as a whole-brain neurodegenerative disorder similar to senile dementia (Alzheimer's disease), but affecting younger individuals. Consequently, extensive research was devoted to characterizing patients "neurological" as well as psychological functions [2].

One type of test device used at that time was the "CronKraepelin" apparatus, which presented letters written on a rotating drum through a $2-\mathrm{mm}$ aperture (Fig. 1A). With this apparatus, the length of stimulus presentation could be controlled by altering the rotation speed of the drum. With this device,
Kraepelin and colleagues were able to demonstrate significant deficits in detection of briefly presented stimuli. However, the need for the drum to complete full slow rotations limited the utility of the device.

A technological advance occurred with the development of an advanced electromechanical apparatus - the "schußplatte" (Fig. 1B) - that allowed presentation of stimuli for as short as 1$\mathrm{ms}$, and was easier to apply than the rotating drum. As with the rotating drum experiments, subjects were asked to identify briefly presented letters. The experiment by Busch using this apparatus was published in 1908 [3] in Kraepelin's journal (reproduced in Supplemental Material), and contains sufficient subject-level data to permit modern re-analysis.

The study included 17 patients diagnosed as dementia praecox, and 6 healthy individuals. Stimuli were presented with a duration of 16.7 ms. Percent correct detections were measured at 0,10 and $30 \mathrm{~s}$ following presentation and summarized per subject. Although statistical analysis was not conduced at the time, in modern terms, the effect-sizes between groups were extremely large $(\mathrm{d}>1.0)$ and highly significant even in the $0 \mathrm{~s}$ delay condition (Fig. 1C).

Although Bleuler stated that his group was unable to substantiate Kraepelin and colleagues' findings, no experimental methods or data were presented. Thus, the quality of the replication study cannot be assessed. Deficits in early visual processing were not rediscovered until the studies of Holzman and colleagues starting in the early 1970's that showed impaired smooth pursuit during eye-tracking, followed by the studies of Braff and colleagues demonstrating increased critical stimulus duration and "transient" visual system dysfunction in schizophrenia (rev. in Ref. [4]).

In modern studies, as in the initial Busch/Kraepelin experiment, schizophrenia patients show reliable, highly significant increases in time required for visual object recognition ("critical stimulus duration") especially to stimuli biased towards the magnocellular visual pathway [5]. This pathway provides critical input into the dorsal stream (often referred as the "where" pathway or "vision for action" system), which is particularly important for the rapid detection of low contrast, low spatial frequency and motion features of stimuli, for processing of information within peripheral visual fields, and for rapidly guiding eye movements towards salient features of the environment [4].

Unlike the alternative "vision for identification" system, individuals are not consciously aware of the functioning of their 
A.
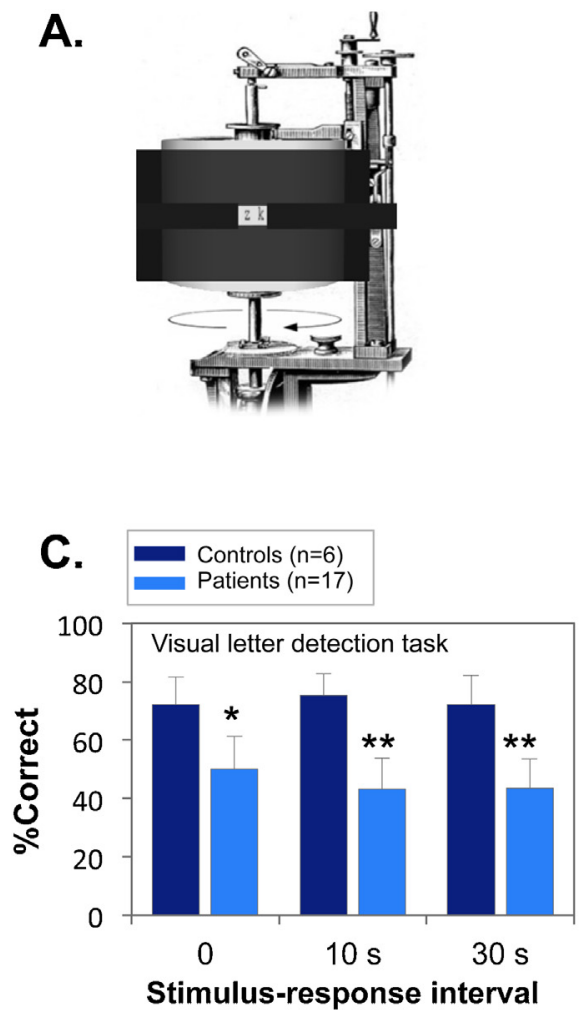

B.
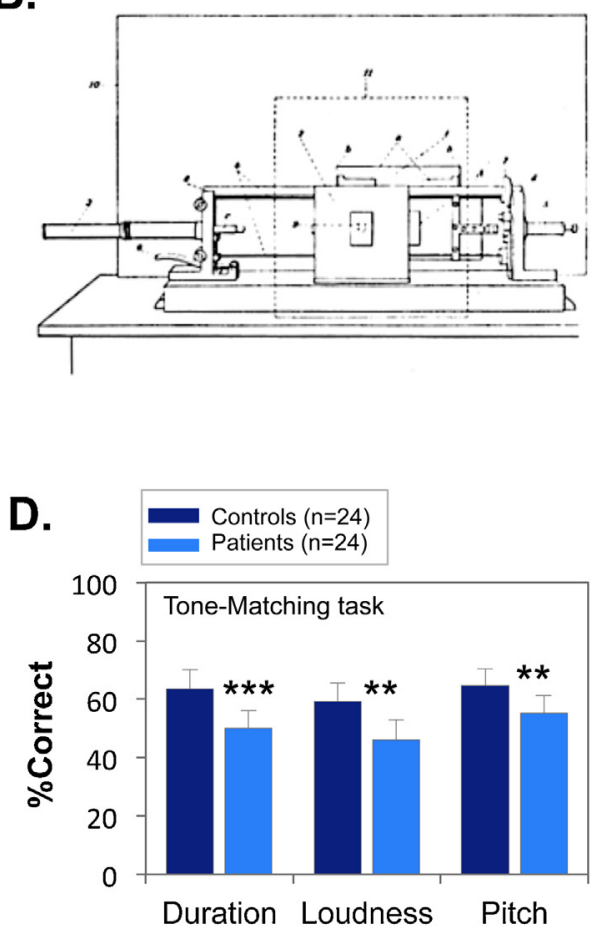

Fig. 1. A. Rotating-drum. (image from: Zimmermann. 1928, S. 185: nachbearbeitet von (c) 2009 H. Maxiilian Wontora).

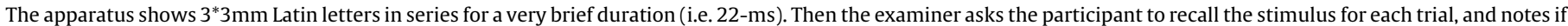
the response is correct or not.

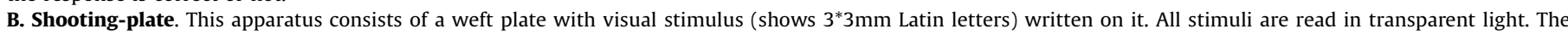

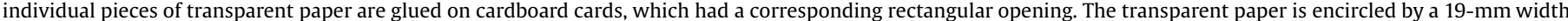

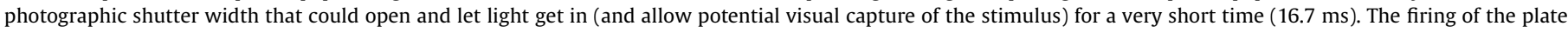

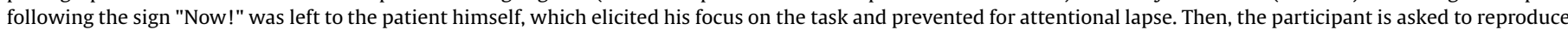
what he saw after breaks of 0,10 and $30 \mathrm{~s}$ in order to gain a measure of his ability to catch and remember visual stimulus after a very-short presentation.

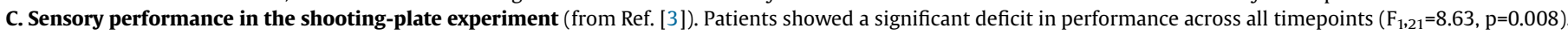
${ }^{*} \mathrm{p}<.05$, between group t-test; ${ }^{* *} \mathrm{p}<.01$.

D. Tone-matching performances (from Ref. [7]). ${ }^{* *} \mathrm{p}<.01$ between groups; ${ }^{* * *} \mathrm{p}<.001$.

magnocellular system. For example, in response to peripheral motion, eye movements may be initiated even before a person is consciously aware of having seen the stimulus. Thus, while some patients spontaneously report visual changes during the early stages of their illness [4], most subjects with magnocellular dysfunction are simply unaware of their visual deficit.

\subsection{Auditory sensory deficits}

As opposed to the visual experiment of Busch and Kraepelin in the early 1900's, reproducible auditory studies of schizophrenia did not become practical until the introduction of commercial tape recorders in the 1950's. In 1965, Billingberg \& Jonsson investigated emotional processing in schizophrenia by testing the hypothesis that patients would misidentify neutral or friendly words as threatening [6]. They administered an "Intonation test", in which single-words were presented with threatening, friendly or neutral intonation.

To control for the more basic ability to identify sounds, an auditory test ("sound effect test") was also included in which participants had to identify a short sound (e,g.., a dentist drill, a train). As predicted, all patients demonstrated paranoid misattribution (i.e. "friendly/neutral" considered as "threatening") significantly more than controls. However, schizophrenia patients were also impaired in the "sound effect test", suggesting the possibility of a more basic underlying auditory deficit.
Consequently, a second study was conducted [7] with an even simpler sensory control task (tone discrimination), in which tonepairs were presented with either identical tones or tones that differed by an acoustic feature (length, intensity or pitch). In this study, there was no significant tendency for patients to misinterpret neutral words as threatening. Nevertheless, deficits were observed in the tone discrimination tasks across a range of physical features (Fig. 1D).

Although these studies were rarely cited at the time of publication, intercorrelated deficits in tone matching and auditory emotion recognition have now been extensively replicated in schizophrenia, and shown to correlate with impaired social function. Auditory sensory deficits have also been demonstrated neurophysiologically using preattentive auditory evoked potentials such as mismatch negativity (MMN), which is robustly impaired in schizophrenia and may predict conversion in clinical high-risk individuals, but is not yet available in clinical practice $[4,8]$.

\section{Sensory deficits in schizophrenia today}

Deficits in sensory processing are now well-established in schizophrenia, and include impairments not only in visual and auditory processing, but also in olfactory and sensorimotor systems. The renewed interest in sensory measures converges with a shift back to the initial Kraepelinian conceptualization of schizophrenia, which views schizophrenia as a "whole brain" disorder. In parallel, these theories converge with neurochemical 
theories focusing on neurotransmitters such as glutamate and GABA that are widely distributed throughout the brain, including within subcortical and cortical sensory regions (rev. in Ref [4]).

To date, no clinical tests are available to capture these deficits. For example, widely used neurocognitive batteries such as the MATRICS Consensus Cognitive Battery (MCCB) use primarily pencil-and-paper testing, and do not evaluate sensory functions. Similarly, eye charts measure primarily ocular pathology, and are not sensitive to magnocellular system dysfunction. Similarly, routine audiometric testing evaluates the ability to detect discrete tones, but not the ability to differentiate between them, and thus is sensitive primarily to middle/inner-ear and brainstem pathology. Therefore, some sensory deficits may be misattributed to "disturbances of attention and higher interest", as was the case during Bleuler's time.

Until tests are available to assess sensory functions routinely within clinical settings, the degree to which sensory deficits contribute to impaired function for any given subjects cannot be easily evaluated. Nevertheless, care must be taken against assuming that because patients can read an eye chart that all aspects of visual perceptual function are intact, or that because they have normal audiometric function that all aspects of auditory function are intact. For example, it has recently been observed that schizophrenia patients with tone-matching deficits benefit from cognitive remediation only if auditory training is also included, while for patients without such deficits, no sensory-based training is required [9].

\section{Conclusions}

In summary, while descriptions of abnormal sensory processing were documented early in the history of schizophrenia, the experimental support at the time was not sufficiently robust to influence pathophysiological conceptualizations of schizophrenia. The basis for the conflicting findings between Kraepelin and Bleuler remains a historical mystery. However, Bleuler's voice rang louder, with influential statements that championed intact sensory processing in schizophrenia holding firm until the early 1970s.

Modern literature makes it increasingly apparent that schizophrenia patients do indeed display specific sensory/perceptuallevel impairments particularly within the visual and auditory sensory systems, and that such deficits may predate illness onset $[4,10]$. At present, as at the time of Kraepelin and Bleuler, the types of deficits observed in schizophrenia are not captured by clinically available optometric and audiometric testing. However, the increasing use of computer- and tablet-based assessment in clinical care settings makes it feasible to start to implement these procedures during routine clinical care, and to consider these deficits when developing personalized treatment approaches.

\section{Acknowledgements}

We would like to acknowledge the assistance of Prof. HansJürgen Moeller in manuscript preparation. Supported by grants MH49334 and MH109298 to DCJ and Fondation de l'AvenirBO-RM18-001 to CD.

\section{Appendix A. Supplementary data}

Supplementary material related to this article can be found, in the online version, at doi:https://doi.org/10.1016/j.eurpsy.2019.04.006.

\section{References}

[1] Bleuler E. Dementia Praecox of the Group of the Schizophrenias. New York, NY: International Universities Press; 1950.

[2] Engstrom EJ, Weber MM. The directions of psychiatric research by Emil Kraepelin. Hist Psychiatry 1887;2005(16):345-64.

[3] Busch A. Auffassungs- und Merkfähigkeit bei Dementia praecox. Psychologische Arbeiten, hrsg v Emil Kraepelin, Band 5. Leipzig: Wilhelm Engelmann Verlag; 1908. p. 293-337.

[4] Javitt DC. When doors of perception close: bottom-up models of disrupted cognition in schizophrenia. Annu Rev Clin Psychol 2009;5:249-75.

[5] Schechter I, Butler PD, Silipo G, Zemon V, Javitt DC. Magnocellular and parvocellular contributions to backward masking dysfunction in schizophrenia. Schizophr Res 2003;64:91-101.

[6] Billingberg $\mathrm{O}$, Jonsson CO. The ability of schizophrenic patients to interpret intonation. Acta Psychiatr Scand 1965;41:218-26.

[7] Jonsson CO, Sjostedt A. Auditory perception in schizophrenia: a second study of the Intonation test. Acta Psychiatr Scand 1973;49:588-600.

[8] Donde C, Luck D, Grot S, Leitman DI, Brunelin J, Haesebaert F. Tone-matching ability in patients with schizophrenia: a systematic review and meta-analysis. Schizophr Res 2017;181:94-9.

[9] Medalia A, Saperstein AM, Qian M, Javitt DC. Impact of baseline early auditory processing on response to cognitive remediation for schizophrenia. Schizophr Res 2019, doi:http://dx.doi.org/10.1016/j.schres.2019.01.012.

[10] Martinez A, Gaspar PA, Hillyard SA, Andersen SK, Lopez-Calderon J, Corcoran $\mathrm{CM}$, et al. Impaired motion processing in schizophrenia and the attenuated psychosis syndrome: etiological and clinical implications. Am J Psychiatry 2018; 175:1243-54.

Clément Dondéa,b,c,* a INSERM, U1028, CNRS, UMR5292, Lyon Neuroscience Research Center, Psychiatric Disorders: from Resistance to Response Team, Lyon, F-69000, France

${ }^{b}$ University Lyon 1, Villeurbanne, F-69000, France

${ }^{c}$ Centre Hospitalier Le Vinatier, Bron, France

Michael Avissar

Dept. of Psychiatry, Columbia University Medical Center, New York, NY USA

Matthias M. Weber Ludwig-Maximilians-Universität München, Medical Faculty, Germany

Daniel C. Javitt ${ }^{\mathrm{a}, \mathrm{b}, * *}$ ${ }^{a}$ Dept. of Psychiatry, Columbia University Medical Center, New York, NY USA

${ }^{b}$ Nathan Kline Institute, Orangeburg, NY USA

* Corresponding author at: Clément Dondé CH Le Vinatier, Batiment 416, 95 boulevard Pinel, BP 300 39, 69678 BRON cedex, FRANCE.

** Corresponding author at: Division of Experimental Therapeutics, Department of Psychiatry, Columbia University Medical Center, 1051 Riverside Drive, New York, NY 10032; United States. E-mail addresses: clement.donde-coquelet@ch-le-vinatier.fr (C. Dondé), dcj2113@cumc.columbia.edu (D. Javitt). Received 22 April 2019 Available online 10 May 2019 